\title{
EI test de proporcionalidad y la calificación del peligro de fuga para dictar prisión preventiva
}

\section{The proportionality test and the rating of the risk of escape to dictate preventive Prison}

\author{
Yoli Marleni Yrigoin Herrera ${ }^{1}$
}

\section{RESUMEN}

El presente trabajo de investigación tuvo como objetivo determinar la aplicación del test de proporcionalidad en la calificación del peligro de fuga para dictar la prisión preventiva en los Juzgados de Investigación Preparatoria de Chachapoyas, 2019, región Amazonas, Perú. La investigación fue de tipo cualitativa, de diseño no experimental, transaccional descriptivo y correlacional. Para la recolección de datos se utilizó la ficha de recojo documental. La muestra estuvo conformada por 19 resoluciones que resuelven dictar prisión preventiva. De los resultados se ha obtenido que, en el $100 \%$ de las resoluciones analizadas si se ha motivado los criterios del arraigo (domiciliario, familiar, laboral y la facilidad de fuga) al momento de dictar la prisión preventiva. Respecto al presupuesto de obstaculización se obtuvo que el $63 \%$ fueron motivadas con el presupuesto de obstaculización para que se dicte la prisión preventiva y en un $37 \%$ no fue motivado éste presupuesto. Se concluye que del $100 \%$ de las resoluciones analizadas mediante las cuales se dicta la prisión preventiva no fueron motivadas en el test de proporcionalidad en sus tres sub principios.

Palabras clave: Proporcionalidad, peligro de fuga

\begin{abstract}
The present research work aimed to determine if the proportionality test is applied in the qualification of the escape danger to dictate preventive detention in the Preparatory Investigation Courts of Chachapoyas, 2019. The research was qualitative type, of non-experimental design, descriptive and correlational transactional. For data collection, the document collection form was used. The sample was made up of nineteen resolutions that resolve to issue preventive detention. From the results, it has been obtained that, $100 \%$ of the resolutions analyzed if the criteria of arraigo (domiciliary, family, labor and ease of escape) have been motivated at the time of issuing the preventive detention. Regarding the obstruction budget, it was obtained that $63 \%$ were motivated with the obstruction budget, so that preventive detention is issued and in $37 \%$ this budget was not motivated. $100 \%$ of the resolutions analyzed by which preventive detention is issued were not motivated in the proportionality test in its three sub-principles.1 Hospital.
\end{abstract}

Keywords: Proportionality, risk of escape

\footnotetext{
${ }^{1}$ Bachiller y Abogada en Derecho y Ciencias Políticas de la Universidad Nacional Toribio Rodríguez de Mendoza de Amazonas. Bachiller y Licenciada en Educación Primaria de la Universidad Nacional Toribio Rodríguez de Mendoza de Amazonas. Egresada de la Maestría en Derecho Constitucional y Administrativo de la Universidad Nacional Toribio Rodríguez de Mendoza de Amazonas: Correo electrónico: yrigoinherrera@gmail.com.
} 


\section{INTRODUCCIÓN}

En el mundo entero, existen miles de personas dentro de las cárceles sin haber sido sentenciadas, en algunas ocasiones por meses, en otras por años, a la espera de un juicio. Las sociedades garantizan derechos, sin embargo preocupa que, ante la ley, los derechos a la seguridad, libertad e igualdad constituyen el soporte de los sistemas judiciales en casi todo el mundo, en las detenciones previas a juicio se emplea una tasa de dos hasta cinco veces mayor en comparación al promedio internacional, por lo que se teme que su uso continúe libremente creciendo. (Aborn y Cannon, 2013)

Del Río (2016), señala que la Corte Constitucional ha establecido como jurisprudencia una adecuada interpretación de la prisión preventiva respetando el principio de presunción de inocencia, ya que esta, solo puede ser aplicada para fines cautelares a fin de asegurar el desarrollo del proceso penal y el eventual cumplimiento de una sanción penal, siempre que exista la certeza de que se evitan los riesgos de fuga y se entorpece el proceso seguido para llegar a la verdad.

El estudio de Almeyda, (2017) sobre la prisión preventiva y el principio de proporcionalidad ha concluido que no se aplica adecuadamente el principio de proporcionalidad por los operadores jurídicos, en las audiencias de prisión preventiva. Por un lado, el fiscal confunde la proporcionalidad de la medida con la proporcionalidad de la pena. Los abogados de la defensa técnica no conocen los subprincipios del test de proporcionalidad, ni lo desarrollan o aplican adecuadamente al caso concreto, ni conocen de técnicas de litigación oral para desarrollar la proporcionalidad de la medida.

El ensayo elaborado por Valle (s.f.) "Importancia del principio de proporcionalidad en la Prisión Preventiva" dentro del cual el autor aborda el tema desde dos puntos de vista: en primer lugar, analiza la constitucionalidad de la prisión preventiva en relación con los derechos fundamentales reconocidos en favor del imputado por la Carta Magna; y, en segundo lugar, la manera como debe aplicarse el principio de proporcionalidad en la prisión preventiva. "En el sistema procesal peruano la prisión preventiva no es de última ratio, sino la "prima ratio", es el principal factor para la existencia de la sobrepoblación carcelaria, que es otro problema de nuestro medio. Por lo que, el test de proporcionalidad es un medio a través del cual se llegaría a controlar el excesivo uso de la prisión preventiva y a su vez sería un medio por el cual las personas sometidas a un proceso penal no sean encarceladas lo que ayudaría a superar esa sobrepoblación carcelaria."

El artículo de Morales denominado "Proporcionalidad de la prisión preventiva. Postura de la Cámara de Apelaciones de Mendoza respecto al requisito de la proporcionalidad de la prisión preventiva", la autora presenta un comentario a un caso de imposición de prisión preventiva que fue revocado por la Cámara de apelaciones al considerar que no era proporcional, fundamentando que "La adopción de esa medida cautelar requiere un juicio de proporcionalidad entre aquélla, los elementos de convicción para dictarla y los hechos que se investigan. Si no hay proporcionalidad, la medida será arbitraria.

La prisión preventiva es una medida cautelar y no punitiva. Se infringe la Convención cuando se priva de libertad, durante un período excesivamente prolongado, y por lo tanto desproporcionado, a personas cuya responsabilidad criminal no ha sido establecida. Esto equivale a anticipar la pena" (Caso López Álvarez Vs. Honduras, Sentencia de 1 de febrero de 2006, Fondo, Reparaciones y Costas, Considerando 68 y 69 )

\section{MATERIAL Y MÉTODO}

La investigación fue de tipo cualitativa, el diseño de la presente investigación fue no experimental, transaccional descriptivo y correlacional. La población estuvo conformada por 19 resoluciones mediante las cuales se dictó prisión preventiva en los Juzgados de Investigación preparatoria de Chachapoyas, región Amazonas, Perú en el año 2019, la población muestral estuvo constituido por 19 resoluciones.

Se utilizó como instrumento la ficha documental y como técnica el análisis documental de las resoluciones judiciales que dictaron prisión preventiva respecto a los presupuestos como el arraigo, el comportamiento del imputado durante el desarrollo del proceso penal, asimismo el análisis respecto a la motivación del test de proporcionalidad en sus tres subprincipios.

\section{RESULTADOS}

Tabla 1

Motivación de los criterios del arraigo para dictar laprisión preventiva

\begin{tabular}{|c|c|c|c|}
\hline $\begin{array}{l}\text { Criterios } \\
\text { arraigo }\end{array}$ & del & Cantidad & Porcentaje \\
\hline Sí & & 19 & $100 \%$ \\
\hline No & & 0 & $0 \%$ \\
\hline Total & & 19 & $100 \%$ \\
\hline
\end{tabular}

De la tabla 1 , se advierte que, en el $100 \%$ de las resoluciones analizadas si se ha motivado los 
criterios del arraigo (arraigo domiciliario, familiar, laboral y la facilidad de fuga) al momento de dictar al prisión preventiva.

\section{Tabla 2}

Motivación del presupuesto de obstaculización al momento de dictar prisión preventiva.

\begin{tabular}{lccc}
\hline $\begin{array}{l}\text { Presupuesto } \\
\text { obstaculización }\end{array}$ & de & Cantidad & Porcentaje \\
\hline Si & 12 & $63 \%$ \\
No & 7 & $37 \%$ \\
\hline & Total & $\mathbf{1 9}$ & $\mathbf{1 0 0 \%}$ \\
\hline
\end{tabular}

De la tabla 2, se advierte que del $100 \%$ de las resoluciones analizadas, en un $63 \%$ se ha motivado el presupuesto de obstaculización del proceso para dictar la prisión preventiva $y$, en un $37 \%$ no se ha motivado.

\section{Tabla 3}

Motivación del test de proporcionalidad, en sus tres sub principios para dictar la prisión preventiva.

\begin{tabular}{lcccc}
\hline & \multicolumn{2}{c}{ Cantidad } & \multicolumn{2}{c}{ Porcentaje } \\
$\begin{array}{l}\text { Test de } \\
\text { proporcionalidad }\end{array}$ & Sí & No & Sí & No \\
\hline Idoneidad & 0 & 19 & $0 \%$ & $100 \%$ \\
Necesidad & 0 & 19 & $0 \%$ & $100 \%$ \\
$\begin{array}{l}\text { Proporcionalidad en } \\
\text { sentido estricto }\end{array}$ & 2 & 17 & $20 \%$ & $80 \%$ \\
\hline Total & \multicolumn{1}{c}{$\mathbf{1 9}$} & $\mathbf{1 0 0 \%}$ \\
\hline
\end{tabular}

De la tabla 3, se advierte que del 100\% de las resoluciones analizadas, ninguna se encuentra motivada con el test de proporcionalidad en sus tres sub principio.

\section{DISCUSIÓN}

Del análisis de las resoluciones judiciales que dictaron prisión preventiva, emitidas por los Juzgados de Investigación Preparatoria de Chachapoyas, 2019, se encontró que del 100\% de las resoluciones si se ha motivado los criterios del arraigo al momento de evaluar y dictar la prisión preventiva, estos criterios son: arraigo domiciliario, arraigo familiar, arraigo laboral y facilidad de fuga; tales resultados difieren con la investigación realizada por Callupe, Palacios \& Polo (2015) en la ciudad de Huánuco, quienes han concluido que los jueces encargados de resolver las prisiones preventivas en dicho departamento, aplican incorrectamente los requisitos establecidos en el CPP, pues en muchas ocasiones las partes acreditaron, con medios probatorios, tener arraigo domiciliario; pese a que, la carga de la prueba en estos casos la tendría el Ministerio Público, no obstante a ello, el juzgado encargado no ha valorado dichas instrumentales, concernientes en certificados domiciliarios, partidas de nacimiento de sus hijos, entre otros.

Por otro lado, los resultados de tabla 1 si guardan relación con lo sostenido por el doctrinario Chirinos (2016), que en el arraigo domiciliario es importante verificar los vínculos familiares, amicales y el arraigo residencial propiamente dicho; situación que también ha sido desarrollada por la Corte Interamericana de Derechos Humanos (2013) en su "Informe sobre el uso de la prisión preventiva en las Américas" cuando establece que, diversos países han ampliado la aplicación de la prisión preventiva introduciendo causales en sus normas procesales de carácter punitivo, no obstante, es necesario tener en cuenta, los criterios tradicionales como el domicilio y la residencia habitual, para cada caso.

Asimismo, nuestros resultados de la tabla 1 guarda relación con lo pronunciado jurisprudencialmente en nuestro país, donde se ha establecido que, el arraigo familiar se considera como el lugar de residencia de los familiares del investigado y el arraigo laboral se encuentra reflejado en la capacidad de subsistencia del imputado en base a un trabajo desarrollado por él dentro del país (Casación $N^{\circ}$ 631-2015 Arequipa, 2015), y que si existiera un arraigo familiar de «mediana intensidad», sería necesario aplicar una medida menos intensa que la de prisión preventiva (Casación No 1445-2018 Nacional, 2019). Por su parte, San Martin (2015) ha dado a conocer que el arraigo familiar del investigado se encontraría demostrado con los lazos familiares de aquel, con la ubicación del domicilio de aquellos y su vínculo con el investigado, lo que acreditaría una unidad familiar.

Además, los resultados obtenidos en la tabla 1 concuerda con lo manifestado por el doctrinario Chirinos (2016), cuando indica que en este punto es necesario tener en cuenta los lazos familiares que el imputado pueda tener en el exterior, así como la doble nacionalidad del agente; circunstancias que también fueron recogidas por San Martin (2015) quien además agregó que es muy importante analizar la situación económica del imputado, ello en cuanto a su solvencia económica, las mismas que permiten que el investigado abandone el país, con mayor facilidad que un imputado que solamente tiene una choza donde vivir; asimismo añade que a esta situación se le debe agregar el hecho que el imputado tenga múltiple nacionalidad, lo cual facilita al abandono del país.

Al realizar el análisis respecto al requisito de la obstaculización, de los resultados tabla 2 se ha obtenido que del $100 \%$ de las resoluciones 
analizadas, en un $63 \%$ se ha motivado el presupuesto de obstaculización al momento la prisión preventiva y en un $37 \%$ no se ha motivado; en tal asentido, de dichos resultados se tiene que no todas las resoluciones fueron motivadas respecto a éste requisito; resultados que concuerdan en cierta medida con la investigación de Vargas (2017), en su tesis realizada en Puno, sobre la debida motivación que deben de tener las resoluciones de prisión preventiva, concluyó que para el año 2015, más de $50 \%$ de resoluciones de prisión preventiva no estaban debidamente motivadas por el juez penal del Segundo Juzgado de Investigación Preparatoria de Puno, a diferencia del año 2016, en donde más del $50 \%$ de las mencionadas resoluciones se encontraban debidamente motivadas.

Finalmente, realizando un análisis respecto a la motivación del test de proporcionalidad, en sus tres sub principios para dictar la prisión preventiva se obtuvo que del 100\% de las resoluciones analizadas, ninguna resolución se encuentra motivada con el test de proporcionalidad en sus tres sub principio: idoneidad, necesidad y proporcionalidad en sentido estricto (ver tabla 3). Resultados que tienen relación con la investigación de Urtecho (2017), en su tesis realizada en el departamento de Ancash, concluyó que la motivación de las resoluciones judiciales del mandato de detención preventiva «es deficiente y limitada; toda vez que dichas resoluciones no cumplen con las exigencias y parámetros de la garantía constitucional de la debida motivación incurriendo mayormente en motivaciones deficientes y aparentes». (p. 156).

Por otro lado, nuestros resultados de la tabla 3 difieren con lo fijado por el Tribunal Constitucional que estableció, "en los casos que conlleven a la restricción de derechos fundamentales, la motivación debe ser superior. Postura además, que se sustenta en el artículo 139 numeral 5) de la Constitución y artículo VI del Título Preliminar del Código Procesal Penal, mismos que establecen que toda resolución que conlleve a una afectación de los derechos tienen que ser debidamente motivada y fundamentada, con especial atención en el caso de las medidas cautelares dictadas contra la persona como es el caso de la prisión preventiva, la misma que por su naturaleza obliga a realizar una fundamentación de mayor intensidad".

Así también, respecto a la proporcionalidad de la medida, nuestros resultados difieren con lo prescrito en el artículo $203^{\circ}$ del Código Procesal Penal que precisa "que las medidas que disponga la autoridad (...) deben realizarse con arreglo al principio de proporcionalidad, y en la medida que existan suficientes elementos de convicción. La resolución que dicte el Juez de Investigación Preparatoria debe ser motivada al igual que el Requerimiento del Ministerio Público"; además, el referido artículo en su inciso 2) expresamente establece que "los
Requerimientos del Ministerio Público serán motivados y debidamente sustentados". Normativa que guarda coherencia con el inciso 2) artículo $253^{\circ}$ del Código Procesal Penal, que a la letra dice "que la restricción de un derecho fundamental requiere expresa autorización legal y se impondrá con respeto del Principio de Proporcionalidad".

De la misma manera, respecto a los resultados de la tabla 3 se tiene que no concuerdan con la investigación de Alegría, Conco, Córdoba \& Herrera, (2011) quienes concluyen que, el principio de proporcionalidad en la regulación de la prisión preventiva es la pieza clave a efecto de que sea la medida que equilibre la necesidad de mantener y respetar el orden social, con el derecho y el respecto a la libertad y el ámbito personal del imputado. La medida que respete este principio debe ser idónea, necesaria y proporcional en sentido estricto; esto es, que supone llevar a cabo un juicio de ponderación en el caso concreto, entre la gravedad o intensidad de la intervención y el peso de las razones que la justifican. En la prisión preventiva, si se respetan las exigencias de idoneidad e intervención mínima, habrá de afirmarse la debida correspondencia de la medida acordada con los elementos que la fundamentan (el "riesgo de frustración" y la "peligrosidad procesal" del imputado): la conformidad del todo con las partes que lo componen que es, al fin y al cabo, el sentido adjetivo "proporcional". Siendo que, para el dictado de dicha medida se ha de verificar si su objeto posibilita que se cumplan con los fines constitucionalmente perseguidos por el proceso penal, si es el medio más idóneo para asegurar su cumplimiento y si es la última ratio del sistema en aquellos casos en donde es ostensible que la libertad del acusado implica un peligro procesal."

Siguiendo con los resultados de la tabla 3 , se observa que no cumple con lo concluido en la investigación de Salvatierra (2018), quien considera que el principio de proporcionalidad se ha convertido en el principal instrumento con el que cuenta el operador jurídico para determinar si la actuación estatal deber tener cobertura constitucional por no ser arbitraria y ajustarse del contenido constitucional de los derechos fundamentales. Y si dicha medida, se ajusta a las exigencias de idoneidad, necesidad, proporcionalidad en sentido estricto y de respeto al contenido constitucionalmente protegido del derecho fundamental en concreto.

\section{CONCLUSIONES.}

Del análisis de las resoluciones que dictan la prisión preventiva se advirtió que, el $100 \%$ de las resoluciones si fueron motivadas respecto a los criterios del arraigo domiciliario, arraigo familiar, arraigo laboral y facilidad de fugas, para dictar la prisión preventiva. 
Respecto al presupuesto de obstaculización se obtuvo que el $67 \%$ de las resoluciones si fueron motivadas y el $37 \%$ no fue motivada con este presupuesto.

En cuanto a la motivación del test de proporcionalidad, en sus tres sub principios (idoneidad, necesidad y proporcionalidad en sentido estricto), el $100 \%$ de las resoluciones mediante las cuales se dictó prisión preventiva no se motivó con el test de proporcionalidad; situación que conlleva a una deficiente motivación de las resoluciones judiciales, ya que está en juego la intervención a los derechos fundamentales de los procesados.

\section{REFERENCIAS BIBLIOGRÁFICAS}

Almeyda, F. (2017). La prisión preventiva y el principio de proporcionalidad en el Distrito Judicial de Cañete 2016. Archivo digital. http://repositorio.ucv.edu.pe/bitstream/han dle/UCV/7513/Almeyda_CFT.pdf?sequen ce $=1 \&$ isAllowed $=y$

Alegría, J, Conco, C, Córdova, J \& Herrera, D. (2011) "El principio de proporciona lidad en materia penal”.

Código Procesal Penal (2017) Lima: Juristas Editores.

Constitución Política del Perú (2019). Lima: Juristas Editores.

Casación N. ${ }^{\circ}$ 631-2015 AREQUIPA (Sala Penal Transitoria de la Corte Suprema de Justicia de la República 21 de Diciembre de 2015).

Casación N. ${ }^{\circ}$ 1445-2018/NACIONAL. Prisión preventiva y peligro de fuga, (Sala Penal Permanente de la Corte Suprema de Justicia de la República 11 de Abril de 2019).

Callupe, L; Palacios, M \& Polo J. (2015). El peligro de fuga como fundamento de la prisión preventiva, vulnera la presunción de inocencia.

Chirinos, J. (2016). Medidas cautelares en el Código Procesal Penal. Lima: IDEMSA.

Del Río, L. (2016). Las medidas cautelares personales del proceso penal peruano. España: Universidad de Alicante. Archivo digital.https://dialnet.uniri oja.es/servlet/ tesis? codigo $=63716$

Morales, X. (s.f.) La prisión preventiva. Postura de la Cámara de Apelaciones de Mendoza respecto al requisito de la proporcionalidad de la prisión preventiva" [Mensaje en un blog]. Archivo digital.http://iaepenal. com/ revista/index.php?option=com content\&vi ew $=$ article \&id=1593\%3 Aproporcionalidad -de-la-prision-preventiva\&catid=301 $\% 3$ Anumero-7\&Itemid=39.

Salvatierra, C. (2018). El test de proporcionalidad y el peligro de su aplicación por el Tribunal Constitucional Peruano. Archivo digital. http://tesis.pucp.edu.pe/repositorio/handle/ 123456789/10271.

San Martin, C. (2015). Derecho Procesal Penal. Lecciones. Lima: Juristas editores.

Urtecho, M. (2017). La debida motivación de las resoluciones judiciales en relación al mandato de detención preventiva y salvaguarda de las garantías del imputado en los juzgados de investigación preparatoria del distrito judicial de Ancash periodo 2012-2013. Archivo digital. http://repositorio. unasam.edu.pe/ bitstream/handle/UNASAM/1653/T033_2 6963922_M.pdf? sequence $=1 \&$ is Allowed $=$ $\mathrm{y}$

Valle, Y. (s.f.) Importancia del principio de proporcionalidad en la Prisión Preventiva. Archivo digital. http://themis.pe/wp/wpcontent/uploads/2016/07/IMPORTANCIADEL-PRINCIPIO-DE PROPORCIONA LID AD-EN-LA PRISI\%C 3\%93NPREVENTIVA-Yunior-Romel-Valle-DeLa-Cruz.docx.

Vargas, Y. (2017). Debida motivación del mandato de prisión preventiva y su aplicación práctica en el Segundo Juzgado de Investigación Preparatoria de la Corte Superior de Justicia de Puno. Archivo digital. http://repositorio. unap.edu.pe/bitstream/ handle/UNAP/4182/Vargas_Ccoya_Ybone _Andrea.pdf? sequence $=1 \&$ isAllowed $=\mathrm{y}$ 